\title{
Estádio de adaptação de Spodoptera frugiperda (J. E. Smith) (Lepidoptera: Noctuidae) em hospedeiros alternativos
}

\author{
Katia Gisele Brasil Boregas (1,2*); Simone Martins Mendes ('); José Magid Waquil (3); Geraldo Wilson \\ Fernandes $\left({ }^{2}\right)$ \\ (') Embrapa Milho e Sorgo, Caixa Postal 285, 35702-098 Sete Lagoas (MG), Brasil. \\ (2) Universidade Federal de Minas Gerais (UFMG). Ecologia Evolutiva \& Biodiversidade/Instituto de Ciências Biológicas, \\ Caixa Postal 483, 30161-970 Belo Horizonte (MG), Brasil. \\ (3) Rede de Inovação Tecnológica Defesa Agropecuária (RIT DA)/CNPq/Consultoria, Belo Horizonte (MG), Brasil. \\ (*) Autora correspondente: kgboregas@gmail.com
}

Recebido: 13/maio/2012; Aceito: 16/abr./2013

\begin{abstract}
Resumo
A principal praga-alvo na cultura do milho é a lagarta-do-cartucho, Spodoptera frugiperda (J.E. Smith, 1797) (Lepidoptera: Noctuidae), que, pela sua ampla distribuição temporal e geográfica constitui-se em uma das espécies mais nocivas nas regiões tropicais das Américas. O objetivo foi avaliar o estádio de adaptação de S. frugiperda em 17 espécies hospedeiras, cultivadas ou selvagens, mais comuns no agroecossistema brasileiro. As plantas foram cultivadas em cinco épocas, entre 2006 e 2008 , utilizando o milho como padrão. Larvas de S. frugiperda recém-eclodidas foram individualizadas e confinadas em copo plástico (50 mL), onde foram alimentadas com seções de folha nova de cada hospedeiro. $O$ alimento foi substituído a cada dois dias por folhas frescas. Quatro variáveis biológicas foram avaliadas e utilizadas para se calcular um Índice de Adaptação (IA). Os resultados indicaram que a sobrevivência inicial de S. frugiperda variou de 100\%, no milho, a 46\%, no arroz. O período larval variou de 12,6 dias, no milho, a 27,1 dias, na grama batatais. A biomassa de pupa variou de 173,1 mg, no carrapicho, a 294,2 mg, no milho. $\mathrm{O}$ índice de adaptação, calculado com base nas variáveis biológicas de S. frugiperda, nos hospedeiros avaliados, variou de 17,43, no milho cultivado na época I, a 1,46, na cana-de-açúcar na época III. Para corrigir o efeito de época sobre o índice de adaptação de S. frugiperda nos hospedeiros, foi calculado o Índice Relativo de Adaptação (IRA), com base no índice de adaptação no milho (100\%). Assim, com base no índice relativo de adaptação, estimado em condições de laboratório, os hospedeiros alternativos ao milho, para S. frugiperda, podem ser assim ordenados, do maior para o menor índice: sorgo granífero, sorgo selvagem, milheto, capim-tanzânia, amendoim, capim-marandu, capim-braquiária, caruru-de-porco, trigo, soja, algodão, feijão, arroz, capim-carrapicho, grama batatais e cana-de-açúcar.
\end{abstract}

Palavras-chave: ecologia, interação inseto-planta, manejo de pragas, lagarta-do-cartucho.

\section{Fitness stage of Spodoptera frugiperda (J. E. Smith) (Lepidoptera: Noctuidae) on alternative hosts}

\section{Abstract}

The main target pest on maize is the fall armyworm, Spodoptera frugiperda (J.E. Smith, 1797) (Lepidoptera: Noctuidae), which, by their seasonal and geographical distribution is one of the most damaging species in tropical regions of America. The objective was the evaluation of S. frugiperda fitness stage on 17 host plant species, cultivated or wild, most common in the Brazilian agro ecosystem. The plants were cultivated in five planting times, between 2006 and 2008, using corn as standard of comparison. Newly hatched larvae of S. frugiperda were individualized and confined in $50 \mathrm{~mL}$ plastic pots, where they were fed with new leaves of each host. Food was replaced every other day with fresh leaf sections of each plant host. Four biological variables were evaluated and used to estimate the Fitness Index (FI). The initial larval survival ranged from 100\% on maize to $46 \%$ on rice. The larval period ranged from 12.6 days on maize to 27.1 days on bahiagrass. The pupa biomass ranged from $173.1 \mathrm{mg}$ on burgrass to $294.2 \mathrm{mg}$ on maize. The fitness index, calculated on the base of S. frugiperda fitness on each host ranged from 17.43 on maize cultivated at time I to 1.46 on sugarcane cultivated at time III. In order to correct the time effect on S. frugiperda fitness stage on hosts, the Relative Fitness Index (RFI) was calculated in relation to maize crop. According to this index, the host rank to S. frugiperda fitness was in decreasing order: grain sorghum, johnson grass, millet, guinea grass, peanut, bread grass, surinam grass, green amaranth, wheat, soybean, cotton, bean, rice, burgrass, bahiagrass and sugarcane. Key words: ecology, insect-plant interaction, pest management, fall armyworm. 


\section{INTRODUÇÃO}

A lagarta-do-cartucho do milho, Spodoptera frugiperda, descrita em 1797 por J. E. Smith, tem ampla distribuição geográfica e ocorre o ano todo em várias culturas como o milho, Zea mays L.; sorgo, Sorghum bicolor (L.) Moench; algodão, Gossypium herbaceum L.; pastagem, Panicum maximum Jacq. Cv. Tanzânia; cana-de-açúcar, Saccharum officinarum L. e soja, Glycine max (L.) Merril. Assim, a $S$. frugiperda constitui-se em uma das espécies mais nocivas para as culturas anuais nas regiōes tropicais das Américas, principalmente no Brasil. As lagartas, tanto no milho, como no sorgo, podem causar perdas de $17 \%$ a $38,7 \%$ na produção, dependendo do ambiente e do estádio de desenvolvimento das plantas atacadas (CRUZ e Turpin, 1983, Williams e Davis, 1984, Cortez e Waquil, 1997, Mendes et al., 2011).

Para o manejo de S. frugiperda em milho, são recomendadas várias estratégias como o uso de plantas transgênicas (milho Bt) associado com manejo de pragas cujo controle químico só deve ser feito quando atingir o nível de ação, o que contribui para a preservação do controle biológico natural. Atualmente tem-se utilizado, basicamente, o milho Bt como único método de controle. Nos sistemas agrícolas convencionais, os efeitos provocados pelas alteraçóes na biodiversidade e no desequilíbrio entre os níveis tróficos tornam o controle da $S$. frugiperda cada vez mais difícil e oneroso. S. frugiperda, por ser polífaga, utiliza vários hospedeiros de ocorrência comum nos diferentes agroecossistemas, os quais contribuem para o aumento de sua densidade populacional em diferentes épocas do ano (WAQuiL et al., 2002). De acordo com o IBGE (2009), na área utilizada com culturas anuais, nos últimos cinco anos, predomina a lavoura de soja (42\%), seguida pela de milho $(29 \%)$ e de feijão (10\%).

Entre as espécies citadas como hospedeiras da $S$. frugiperda, a soja é uma das mais abundantes no agroecossistema, durante o verão. Assim, esta espécie pode ter um papel importante no estabelecimento e na colonizaçáo do ambiente pelas comunidades de insetos herbívoros e de seus inimigos naturais, incluindo $S$. frugiperda, apesar de não ser um de seus hospedeiros preferenciais.

Embora a soja esteja listada como hospedeira de S. frugiperda, esta raramente tem sido citada causando danos na soja. Como a cultura do milho ou sorgo é comumente utilizada em rotação ou sucessão à cultura da soja, esta tem sido intensivamente exposta à pressão populacional de $S$. frugiperda, pela coexistência no mesmo agroecossistema. Assim, é possível inferir que a incidência de $S$. frugiperda na soja pode vir a causar perdas significativas também nessa cultura. Dependendo dos hospedeiros disponíveis no agroecossistema e do desempenho de $S$. frugiperda em cada um desses hospedeiros, alteraçôes significativas podem ocorrer na dinâmica populacional dessa espécie.
Desta forma, o conhecimento do desempenho biológico de $S$. frugiperda, nos principais hospedeiros alternativos, deve contribuir para melhor entendimento da sua ecologia e melhoria dos atuais programas de manejo integrado de pragas nos sistemas de produção envolvendo rotação ou sucessão, bem como para a definição de estratégias para o manejo da resistência de $S$. frugiperda em plantas geneticamente modificadas que, desde 2008, vêm sendo comercialmente utilizadas no Brasil (CTNBio, 2011).

O objetivo deste estudo foi quantificar, em condiçôes de laboratório, o estádio adaptativo de $S$. frugiperda nos principais hospedeiros alternativos encontrados em agroecossistemas envolvendo a cultura do milho no Brasil.

\section{MATERIAL E MÉTODOS}

Os bioensaios foram realizados no laboratório de Ecotoxicologia de Insetos do Centro Nacional de Pesquisa de Milho e Sorgo - CNPMS/Embrapa, em Sete Lagoas (MG), Brasil. Dentre as 148 espécies de plantas hospedeiras de $S$. frugiperda registradas na literatura (Pogue, 1995), foram selecionadas as 17 espécies mais comumente encontradas no agroecossistema, onde se cultiva milho no Brasil (Tabela 1).

Para viabilizar o manejo dos bioensaios, em funçáo da máo de obra disponível e da melhor época do ano para o cultivo, as plantas hospedeiras foram cultivadas no campo, em três épocas, com intervalo de 30 dias. O milho, por ser considerado o hospedeiro preferencial de S. frugiperda (Cruz, 1995; SÁ et al., 2009), foi repetido em todas as épocas, para servir como controle. Os cinco hospedeiros que tiveram resultados contrastantes nas três primeiras épocas foram novamente submetidos a novos bioensaios, em duas outras épocas. Os trabalhos foram realizados entre novembro de 2006 e julho de 2008 , de acordo com o grupo de hospedeiro, nas seguintes épocas (meses/anos): I (11-12/2006), II (3-4/2007), III (7-8/2007), IV (9-10/2007) e V (6-7/2008).

O bioensaio foi iniciado no laboratório, quando as plantas hospedeiras atingiram o estádio de 4-5 folhas, aproximadamente 15 dias após a germinação. Todas as avaliaçôes foram realizadas em laboratório com iluminação natural intensa e em condiçôes controladas de temperatura $\left(26 \pm 2^{\circ} \mathrm{C}\right)$ e umidade relativa $(60 \pm 10 \%)$. Assim, a fotofase foi a mesma em que as plantas hospedeiras estavam expostas no campo durante seu desenvolvimento. Foram avaliadas as seguintes variáveis biológicas:

\section{Sobrevivência larval}

Esta variável foi avaliada em duas etapas, sobrevivência inicial (primeiro ínstar) e sobrevivência durante 
Tabela 1. Lista das espécies de plantas hospedeiras utilizadas para avaliação do índice de adaptação de Spodoptera frugiperda (J. E. Smith). Adaptada de "Spodoptera Database"

\begin{tabular}{|c|c|c|c|}
\hline Nome Científico & Cultivar & Nome comum & Família \\
\hline Amaranthus viridis $L$. & planta invasora & caruru-de-porco & Amaranthaceae \\
\hline Arachis hypogaea L & IAC 886 & Amendoim & Fabaceae \\
\hline Brachiaria brizantha (Hochst) Stapf & Brizantha & capim-marandu & Poaceae \\
\hline Brachiaria decumbens Stapf & Basilisk & capim-braquiária & Poaceae \\
\hline Cenchrus echinatus (L.) & planta invasora & capim-carrapicho & Poaceae \\
\hline Gossypium herbaceum L. & EPMG-Redenção & Algodão & Malvaceae \\
\hline Glycine max (L.) Merril & Valiosa RR & Soja & Fabaceae \\
\hline Oryza sativa L. & BRSMG-Conai & Arroz & Gramineae \\
\hline Panicum maximum Jacq. & Tanzânia & capim-tanzânia & Poaceae \\
\hline Paspalum notatum Fluggé & jardinagem & grama batatais & Poaceae \\
\hline Phaseolus vulgaris L. & Pérola & Feijão & Fabaceae \\
\hline Saccharum officinarum L. & IAC-Forrageira & cana-de-açúcar & Poaceae \\
\hline Sorghum arundinaceum (Willd.)Stapf & planta invasora & sorgo selvagem & Poaceae \\
\hline Sorghum bicolor (L.) Moench & BRS 310 & sorgo granífero & Poaceae \\
\hline Triticum aestivum L. & Embrapa-42 & Trigo & Poaceae \\
\hline Zea mays L. & BRS 1030 & Milho & Poaceae \\
\hline
\end{tabular}

Fonte: Pogue (1995).

todo o período larval. Inicialmente, selecionou-se um número conhecido de ovos (cerca de 50) próximo à eclosão, obtidos de colônia de $S$. frugiperda, coletadas na fase de larva em milho na área experimental da Embrapa Milho e Sorgo e mantida em laboratório por vários anos em dieta artificial no laboratório de entomologia para a infestação. Os insetos foram acondicionados em caixa Gerbox ${ }^{\circledR}(11,0 \times 11,0 \times 3,5 \mathrm{~cm}$ de altura), com seçóes de folhas de cada hospedeiro em tamanho médio de $50 \mathrm{~cm}^{2}$, para avaliar a sobrevivência inicial, ou seja, 48 horas após a eclosão. Para o cálculo da sobrevivência inicial, avaliou-se o número de larvas eclodidas e sobreviventes em cada época de cultivo dos hospedeiros.

Para avaliar a sobrevivência durante todo o desenvolvimento larval e evitar o canibalismo, larvas sobreviventes após as 48 horas, depois de avaliadas, foram confinadas individualmente em cada hospedeiro, utilizando copos plásticos (café), com capacidade de $50 \mathrm{~mL}$ e tampas de acrílico transparente. Os copos foram acondicionados em bandejas de isopor, as quais foram mantidas em estantes de metal. Foram utilizadas cem repetiçóes, sendo cada repetição um copo com uma larva. Sempre foram utilizadas três seçôes de folhas tenras, com cerca de 10 centímetros quadrados, de cada hospedeiro. Os pedaços de folhas foram substituídos a cada dois dias, até completar o período larval ou a morte do inseto, quando também foram anotadas as sobrevivências larvais. Para computar a porcentagem de sobrevivência, cada grupo de dez dos cem avaliados foi considerado uma repetição.

\section{Período larval}

O período de desenvolvimento larval de $S$. frugiperda foi determinado a partir do dia da eclosão das larvas até o primeiro dia em que se observou a pupa. As observaçôes foram realizadas a cada dois dias.

\section{Biomassa de pupas}

A biomassa de cada pupa de $S$. frugiperda, para cada tratamento, foi registrada no primeiro dia de sua observaçáo, utilizando-se balança de precisão $(0,1 \mathrm{mg})$, tomando-se o cuidado para não danificar o inseto durante a operação.

\section{Índice de Adaptação (IA)}

Para estimar o estádio de adaptação de $S$. frugiperda nos 17 hospedeiros, foi utilizado o índice de adaptação. Assim, com um único indicador se pode avaliar e classificar o estádio de adaptação de $S$. frugiperda em cada hospedeiro. Este índice é uma adaptaçáo do Índice de Suscetibilidade (IS) proposto por Pencoe e Martin (1982). O Índice de Suscetibilidade é calculado segundo a fórmula:

\section{$\mathrm{IS}=(\mathrm{SBL} * \mathrm{FDA}) /(\mathrm{PDL} * \mathrm{AFC})$}

Em que: $I S=$ índice de suscetibilidade, $S B L=$ sobrevivência larval, FDA=fecundidade dos adultos, $\mathrm{PDL}=$ período de desenvolvimento larval (dias) e AFC=área foliar consumida. 
O índice de suscetibilidade quantifica tanto o estádio de adaptação do inseto ao hospedeiro, quanto a sensibilidade do hospedeiro aos danos causados pela espécie em questáo. Como o objetivo deste trabalho foi avaliar apenas o estádio de adaptação do inseto nos diferentes hospedeiros, foi eliminado da fórmula de Pencoe e Martin (1982) o consumo foliar, simplificando a fórmula para:

\section{$\mathrm{IA}=(\mathrm{SBL} * \mathrm{FDA}) /(\mathrm{PDL})$}

Em que: $\mathrm{IA}=$ índice de adaptação, $\mathrm{SBL}=$ sobrevivência larval, FDA=fecundidade dos adultos e $\mathrm{PDL}=$ período de desenvolvimento larval.

Como a sobrevivência larval foi estimada em duas etapas, para o cálculo do IA considerou-se a sobrevivência de todo o período larval, multiplicando-se as duas razóes (sobrevivência inicial e a durante o restante do período larval).

A justificativa para alteração da fórmula inicial do índice de suscetibilidade se baseia no fato de que a área foliar consumida afeta principalmente a planta, mas a quantidade consumida pode ser considerada irrelevante para a dinâmica populacional do inseto em condiçáo de abundância do hospedeiro no agroecossistema. Assim, o índice deixa de ser de suscetibilidade, pois passa a usar apenas as variáveis do inseto, passando a indicar apenas o estádio de adaptação deste no hospedeiro. Portanto, pode ser referido apenas como Índice de Adaptação (IA). Como a fecundidade dos adultos em gaiolas é afetada pela condição artificial, ela foi estimada baseando-se na biomassa de pupas. De acordo com Leuck e Perkins (1972), a fecundidade de S. frugiperda é significativamente correlacionada com o peso de pupas. Também para outras espécies, como Antheraea assama Westwood (Lepidoptera: Saturniidae), foi demonstrado por BARAH e Sengupta (1993) que o número de ovos colocados por fêmea está positivamente correlacionado com a massa de pupas $(\mathrm{r}=0,973)$ e significativo ao nível de $1 \%$ de probabilidade. Assim, por ser a biomassa de pupa uma variável relativamente fácil de ser obtida com precisão, ela pode ser usada para estimar a fecundidade dos insetos desenvolvidos nos diferentes hospedeiros.

\section{Índice Relativo de Adaptação (IRA)}

Considerando que as variáveis biológicas estudadas, sobrevivência larval, período larval e biomassa de pupas variam de acordo com as condiçóes ambientais, as quais afetam também os hospedeiros, os dados obtidos nas diferentes épocas de avaliaçáo não podem ser comparados diretamente. Consequentemente, também o índice de adaptação de $S$. frugiperda deve variar entre as épocas. Neste caso, optou-se por se calcular um índice relativo de adaptação para S. frugiperda com base no índice de adaptação obtido no milho, por ser seu hospedeiro preferencial (Cruz, 1995). Como o índice de adaptação deve variar, mesmo entre as diferentes cultivares de milho, em todas as épocas de avaliação, foi utilizado o híbrido BRS 1030 como testemunha e, assim, corrigir o efeito de época sobre o estádio de adaptação de $S$. frugiperda nos diferentes hospedeiros. Considerando que o índice de adaptação de $S$. frugiperda no milho representa o estádio de adaptação mais avançado (100\%), o Índice Relativo de Adaptação (IRA) foi calculado:

$\mathrm{IRA}=100 *(\mathrm{IAh}) /(\mathrm{IAm})$

Em que: IAh=índice de adaptação de $S$. frugiperda no hospedeiro em questão e IAm=índice de adaptação de S. frugiperda no milho avaliado como testemunha em cada bioensaio. O índice relativo de adaptação pode ser expresso em porcentagem ou como um valor absoluto, retirando o fator 100 da equaçáo apresentada acima.

\section{Análise estatística}

Para ajustar os hospedeiros à sua melhor época de desenvolvimento durante o ano e facilitar a avaliação dos 17 hospedeiros, os tratamentos variaram a cada época. A análise foi realizada comparando os tratamentos (hospedeiros) em cada época independentemente. Para todas as variáveis, os dados foram submetidos à ANOVA. Quando houve indicação de diferença significativa, as médias foram comparadas pelo teste de Tukey $(\mathrm{p} \leq 0,05)$.

Para avaliar a correlação de cada uma dessas variáveis biológicas com o índice de adaptação, foram estimados os coeficientes de correlação utilizando o programa estatístico livre SISVAR (Ferreira, 2003). Os dados da sobrevivência inicial e a sobrevivência durante o resto do período larval foram transformados em $(x+0,5)^{1 / 2}$ para efeitos de análise estatística.

\section{RESULTADOS E DISCUSSÃO}

As dezessete espécies de plantas hospedeiras de $S$. frugiperda, selecionadas para este estudo, foram avaliadas inicialmente em três épocas (Tabela 2). Os hospedeiros com resultados contrastantes foram reavaliados em duas outras épocas para confirmar os resultados obtidos anteriormente (Tabela 3).

Nas duas últimas épocas de avaliação, além do milho, o milheto foi repetido, por ter resultados muito semelhantes àqueles encontrados em milho como hospedeiro de S. frugiperda. Nas cinco épocas de avaliação, o milho se destacou como o melhor hospedeiro para $S$. frugiperda, com base na sobrevivência, biomassa de pupas e período de desenvolvimento. Também, para todas as variáveis, foram observadas diferenças significativas entre os 
Tabela 2. Médias ( $\pm \mathrm{EP})$ das variáveis biológicas de Spodoptera frugiperda, cujas larvas foram alimentadas em diferentes hospedeiros cultivados em três épocas. Sete Lagoas (MG)

\begin{tabular}{|c|c|c|c|c|c|c|c|c|c|}
\hline \multirow{2}{*}{ Época* } & \multirow{2}{*}{$\begin{array}{c}\text { Hospedeiro } \\
\text { Arroz }\end{array}$} & \multicolumn{2}{|c|}{$\begin{array}{l}\text { Sobrevivência } \\
\text { inicial (razão) }^{(1)}\end{array}$} & \multicolumn{2}{|c|}{$\begin{array}{c}\text { Sobrevivência larval } \\
\text { (razão) }^{(1)}\end{array}$} & \multicolumn{2}{|c|}{$\begin{array}{l}\text { Período larval } \\
\text { (dias) }\end{array}$} & \multicolumn{2}{|c|}{$\begin{array}{l}\text { Biomassa de } \\
\text { pupas }(\mathrm{mg})\end{array}$} \\
\hline & & $0,46 \pm 0,07$ & c & $0,74 \pm 0,05$ & $a b$ & $16,7 \pm 0,07$ & $\mathrm{a}$ & $213,8 \pm 2,86$ & $b$ \\
\hline I & Feijão & $0,83 \pm 0,04$ & $b$ & $0,60 \pm 0,06$ & $b$ & $14,3 \pm 0,08$ & b & $236,2 \pm 4,95$ & $a b$ \\
\hline I & milheto & $1,00 \pm 0,00$ & $\mathrm{a}$ & $0,77 \pm 0,05$ & $a b$ & $12,9 \pm 0,05$ & c & $227,0 \pm 2,41$ & $a b$ \\
\hline I & Milho & $0,99 \pm 0,05$ & a & $0,88 \pm 0,06$ & a & $12,6 \pm 0,11$ & c & $246,1 \pm 3,28$ & $\mathrm{a}$ \\
\hline I & Soja & $0,73 \pm 0,05$ & $b$ & $0,74 \pm 0,05$ & $a b$ & $15,5 \pm 0,06$ & $\mathrm{a}$ & $241,4 \pm 2,95$ & $\mathrm{a}$ \\
\hline I & Sorgo granífero & $1,00 \pm 0,00$ & $\mathrm{a}$ & $0,85 \pm 0,04$ & $\mathrm{a}$ & $12,8 \pm 0,06$ & c & $244,2 \pm 6,24$ & $\mathrm{a}$ \\
\hline II & Algodão & $0,82 \pm 0,04$ & bc & $0,74 \pm 0,05$ & $a b$ & $17,6 \pm 0,19$ & $b$ & $209,4 \pm 3,99$ & $b$ \\
\hline II & Amendoim & $0,94 \pm 0,02$ & $a b$ & $0,80 \pm 0,04$ & $\mathrm{a}$ & $17,7 \pm 0,08$ & $b$ & $240,3 \pm 3,88$ & a \\
\hline II & Capim-carrapicho & $0,70 \pm 0,06$ & c & $0,61 \pm 0,06$ & b & $20,3 \pm 0,13$ & $\mathrm{a}$ & $173,8 \pm 5,44$ & c \\
\hline II & Capim-braquiária & $0,97 \pm 0,02$ & $a b$ & $0,82 \pm 0,04$ & $\mathrm{a}$ & $16,6 \pm 0,10$ & bc & $201,6 \pm 4,51$ & $b$ \\
\hline II & Capim-marandu & $0,87 \pm 0,04$ & $a b$ & $0,79 \pm 0,05$ & $\mathrm{a}$ & $14,7 \pm 0,10$ & $c d$ & $218,3 \pm 4,13$ & $b$ \\
\hline II & Milho & $0,99 \pm 0,01$ & $\mathrm{a}$ & $0,82 \pm 0,09$ & $\mathrm{a}$ & $12,8 \pm 0,12$ & d & $241,1 \pm 4,10$ & $\mathrm{a}$ \\
\hline III & Cana-de-açúcar & $0,88 \pm 0,03$ & $a b$ & $0,13 \pm 0,09$ & $d$ & $17,4 \pm 1,00$ & $b$ & $221,9 \pm 12,66$ & c \\
\hline III & Caruru-de-porco & $0,99 \pm 0,01$ & $\mathrm{a}$ & $0,97 \pm 0,02$ & a & $22,5 \pm 0,10$ & $a b$ & $191,7 \pm 7,56$ & d \\
\hline III & Grama batatais & $0,81 \pm 0,04$ & $b$ & $0,46 \pm 0,07$ & c & $27,1 \pm 0,11$ & $\mathrm{a}$ & $218,0 \pm 3,46$ & c \\
\hline III & Milho & $1,00 \pm 0,00$ & $\mathrm{a}$ & $0,90 \pm 0,03$ & $a b$ & $18,2 \pm 0,15$ & $b$ & $262,6 \pm 3,76$ & $b$ \\
\hline III & Sorgo selvagem & $0,94 \pm 0,02$ & a & $0,85 \pm 0,04$ & $a b$ & $18,1 \pm 0,15$ & $b$ & $284,6 \pm 3,30$ & $\mathrm{a}$ \\
\hline III & Capim-marandu & $1,00 \pm 0,00$ & $\mathrm{a}$ & $0,77 \pm 0,05$ & $b$ & $16,7 \pm 0,21$ & $b$ & $226,8 \pm 3,67$ & c \\
\hline
\end{tabular}

Médias seguidas da mesma letra, dentro de cada coluna e época, năo diferem entre si, pelo teste de Tukey, a $5 \%$ de probabilidade; $\left({ }^{1}\right)$ Dados transformados em $(\mathrm{x}+0,5)^{1 / 2}$ para submeter à análise estatística; *Épocas (meses/anos): I (11-12/2006), II (3-4/2007), III (7-8/2007).

Tabela 3. Médias $( \pm \mathrm{EP})$ das principais variáveis biológicas de Spodoptera frugiperda, cujas larvas foram alimentadas em hospedeiros selecionados nas três épocas anteriores e cultivados em duas épocas. Sete Lagoas (MG)

\begin{tabular}{|c|c|c|c|c|c|c|c|c|c|}
\hline Época* & Hospedeiro & $\begin{array}{r}\text { Sobrevivência } \\
\text { (razão) }\end{array}$ & inicial & $\begin{array}{r}\text { Sobrevivência } \\
\text { (razão) }\end{array}$ & larval & $\begin{array}{c}\text { Período lar } \\
\text { (dias) }\end{array}$ & & $\begin{array}{l}\text { Biomassa c } \\
\text { pupas }(\mathrm{mg}\end{array}$ & \\
\hline IV & Milheto & $0,90 \pm 0,03$ & $\mathrm{a}$ & $0,78 \pm 0,05$ & b & $15,7 \pm 0,12$ & $\mathrm{a}$ & $247,3 \pm 3,96$ & $a b$ \\
\hline IV & Milho & $0,97 \pm 0,02$ & a & $0,92 \pm 0,03$ & $\mathrm{a}$ & $15,9 \pm 0,06$ & a & $294,2 \pm 3,56$ & $\mathrm{a}$ \\
\hline IV & Trigo & $0,89 \pm 0,03$ & $\mathrm{a}$ & $0,78 \pm 0,05$ & $b$ & $15,9 \pm 0,07$ & $\mathrm{a}$ & $216,3 \pm 5,69$ & $b$ \\
\hline V & Cana-de-açúcar & $0,53 \pm 0,07$ & $\mathrm{~b}$ & $0,49 \pm 0,07$ & $b$ & $24,1 \pm 0,11$ & a & $215,0 \pm 5,06$ & $d$ \\
\hline V & Milheto & $0,88 \pm 0,03$ & a & $0,82 \pm 0,04$ & a & $15,5 \pm 0,06$ & c & $231,6 \pm 2,45$ & c \\
\hline V & Milho & $0,94 \pm 0,02$ & $\mathrm{a}$ & $0,89 \pm 0,03$ & $\mathrm{a}$ & $16,9 \pm 0,03$ & b & $273,9 \pm 2,28$ & $\mathrm{a}$ \\
\hline V & Sorgo granífero & $0,91 \pm 0,03$ & $\mathrm{a}$ & $0,89 \pm 0,03$ & $\mathrm{a}$ & $15,0 \pm 0,01$ & c & $270,9 \pm 2,29$ & $\mathrm{a}$ \\
\hline V & Sorgo selvagem & $0,95 \pm 0,02$ & $\mathrm{a}$ & $0,88 \pm 0,03$ & $\mathrm{a}$ & $15,3 \pm 0,02$ & c & $256,6 \pm 3,38$ & $b$ \\
\hline
\end{tabular}

Médias seguidas da mesma letra não diferem entre si (em cada coluna e época), pelo teste de Tukey, a 5\% de probabilidade; $\left({ }^{1}\right)$ Dados transformados em $(\mathrm{x}+0,5)^{1 / 2}$ para submeter à análise estatística; *Épocas avaliadas: IV (setembro- outubro/2007) e V (junho-julho/2008).

hospedeiros, exceto para a sobrevivência inicial e período larval entre o milho, milheto e trigo (Época IV). Em geral, as variáveis biológicas de $S$. frugiperda estudadas não diferiram significativamente entre o milho, milheto, sorgo granífero e sorgo selvagem.

Resultados semelhantes para a sobrevivência larval, período de desenvolvimento larval e biomassa de pupas, de S. frugiperda alimentada com folhas de milho, sorgo granífero e milheto, em laboratório, foram reportados por SÁ et al. (2009). Segundo Murúa e VirLa (2004), a fertilidade de $S$. frugiperda variou de $92,1 \%$, em milho, 96,4\%, em P. maximum, a 99,4\%, em C. dactylon. Portanto, $S$. frugiperda se adapta bem em vários hospedeiros e estudos mais detalhados precisam ser desenvolvidos para definir que papel o complexo de hospedeiros desempenha na dinâmica populacional dessa espécie polífaga.

\section{Sobrevivência larval}

A sobrevivência larval de $S$. frugiperda foi significativamente diferente entre alguns hospedeiros (Tabelas 2 e 3). A sobrevivência inicial, em todas as épocas, foi maior no milho e acima de $90 \%$. Ela foi significativamente menor no arroz, no carrapicho, na grama batatais e na cana-de-açúcar (época V). A sobrevivência inicial de $S$. frugiperda não diferiu significativamente entre o milho, milheto e sorgo granífero, na época I; entre milho, amendoim, capim-braquiária e capim-marandu, na época II; e entre milho, cana-de-açúcar, caruru-de-porco, sorgo selvagem, capim-tanzânia e trigo, na época III. Durante todo o período larval, observou-se a maior variação da sobrevivência de $S$. frugiperda nos diferentes hospedeiros, do que foi registrado na sobrevivência inicial. A maior sobrevivência larval de $S$. frugiperda foi 
registrada no milho e as menores sobrevivências no feijão, no carrapicho e na cana-de-açúcar. Essa variável não diferiu, significativamente, entre o milho, milheto, sorgo granífero, sorgo selvagem, arroz e soja, na época I; entre milho, algodão, amendoim, capim-braquiária e capim-marandu, na época II; e entre milho, caruru-de-porco e sorgo selvagem, na época III. Embora a cana-de-açúcar seja considerada um hospedeiro de $S$. frugiperda, apenas a sobrevivência inicial na época III foi semelhante à do milho, mas, a sobrevivência durante todo o período larval, nessa época, e a sobrevivência inicial e larval, na época $V$ foram muito baixas. Esse fato se deve, provavelmente, às características físicas da folha, que, além de ser fibrosa, perde umidade facilmente após o corte, dificultando a alimentação pelas lagartas. A alta sobrevivência de $S$. frugiperda em hospedeiros alternativos foi observada também por Pencoe e Martin (1982) e em milho, sorgo, milheto e soja por Sá et al (2009). Considerando um biótipo milho de $S$. frugiperda, MEAGHER et al. (2004) verificaram maior sobrevivência desse biótipo no sorgo selvagem $(95,6 \%)$ do que no próprio milho $(62,2 \%)$. Bavaresco et al. (2003) registraram sobrevivência muito baixa de Spodoptera cosmioides (Walk.) em mamona $(21,7 \%)$, cebola $(21,8 \%)$ e soja $(3,9 \%)$.

\section{Período larval}

O período de desenvolvimento larval de $S$. frugiperda, medido em dias, foi significativamente diferente entre os hospedeiros avaliados em quatro, das cinco épocas estudadas (Tabelas 2 e 3). Apenas na época IV, quando o milho foi comparado com o milheto e trigo, não se observou diferença significativa entre os tratamentos. O período larval de $S$. frugiperda foi táo curto como no milho, nos hospedeiros milheto, sorgo granífero, sorgo selvagem, capim-marandu, capim-tanzânia, caruru-de-porco e na cana-de-açúcar na época III. Por outro lado, na época $V$, o maior período larval de $S$. frugiperda foi observado na cana-de-açúcar. O período larval de $S$. frugiperda foi intermediário no algodáo, amendoim, braquiária, na soja e no feijão, embora, nestes dois últimos, tenha sido significativamente diferente entre eles.

Geralmente, quanto mais curto o período de desenvolvimento larval, sem redução de biomassa, pode-se inferir que mais adequado e melhor é o hospedeiro para o desenvolvimento do inseto. Em laboratório, SÁ et al. (2009) não observaram diferenças significativas no período larval de $S$. frugiperda alimentada com folhas de milho, sorgo, milheto, soja ou braquiária, confirmando os resultados aqui observados, exceto para soja e braquiária que foi significativamente maior do que nos demais. PAnizzi et al. (2004), avaliando em laboratório o desenvolvimento de lagartas de Anticarsia gemmatalis (Hübner), em 17 espécies vegetais, observaram que houve diferenças entre algumas leguminosas. Não houve diferença significativa no período de desenvolvimento larval de $A$. gemmatalis na soja, guandu, caupi e crotalária, mas foi maior nas larvas alimentadas em tremoço azul, ervilhaca e em ervilha. Segundo Moscardi et al. (1981), o alto valor nutricional do hospedeiro acelera o desenvolvimento das larvas.

\section{Biomassa de pupa}

Quando confinada em um recipiente totalmente fechado, a larva de $S$. frugiperda, antes de entrar na fase de pupa, prepara sua câmara pupal com os restos de alimentos, onde permanece até a emergência do adulto. Como houve alta mortalidade de larvas em alguns hospedeiros durante o período de desenvolvimento larval, o número de insetos avaliados variou entre os tratamentos. No milho, a biomassa de pupas de $S$. frugiperda variou de $241,1 \mathrm{mg}$, na época II, a 294, $2 \mathrm{mg}$, na época IV (Tabelas 2 e 3). Entre todas as épocas, apenas as larvas alimentadas com folhas do sorgo selvagem, na época III, tiveram a média da biomassa de pupas significativamente maior do que a das pupas de larvas desenvolvidas no milho. As médias da biomassa de pupas obtidas de larvas alimentadas com folhas de feijão, soja, sorgo granífero e amendoim não diferiram significativamente daquelas desenvolvidas no milho. Por outro lado, a média da biomassa de pupas das larvas alimentadas com folhas de milheto não diferiu da média das desenvolvidas no milho, nas épocas I e IV, mas foi significativamente menor na época $V$. As médias da biomassa de pupas das larvas de $S$. frugiperda alimentadas com folhas do arroz, algodão, capim-carrapicho, capim-braquiária, capim-marandu, cana-de-açúcar, caruru-de-porco, grama batatais, capim-tanzânia e trigo foram, significativamente, menores do que a média da biomassa de pupas desenvolvidas no milho. Embora, na época III, a média da biomassa de pupas obtidas no sorgo selvagem tenha sido significativamente maior do que a média obtida no milho, na época $V$ essa média foi menor. No milheto, nas épocas I e IV, a média da biomassa de pupas das larvas não diferiu significativamente das desenvolvidas no milho, mas foi significativamente menor na época $V$.

Analisando a sobrevivência (inicial e durante o restante da fase larval), o período larval e a biomassa de pupas de S. frugiperda, em cada hospedeiro, verifica-se que apenas no milheto e no sorgo granífero na época I, essas variáveis nâo discriminaram significativamente esses tratamentos do milho (Tabelas 2 e 3). Em contraste, a grama batatais, o arroz e a cana-de-açúcar diferiram do milho em pelo menos três dessas quatro variáveis analisadas. Nas médias dos demais hospedeiros não houve diferenças significativas em duas ou mais das variáveis estudadas. Portanto, com base nessas variáveis analisadas, é possível separar o conjunto estudado em pelo menos três grupos de hospedeiros. Entretanto, fica difícil elaborar um ordenamento dos hospedeiros de $S$. frugiperda, analisando o conjunto dessas variáveis estudadas. Desse modo, foi calculado um índice, ponderando-se todas as variáveis estudadas, sendo 
estimado um valor específico do estádio de adaptação do inseto em cada hospedeiro.

\section{Índice de Adaptação}

Os índices de adaptação de $S$. frugiperda foram calculados, segundo a equação apresentada em Material e Métodos, para cada hospedeiro por época de avaliação e estão apresentados na tabela 4. Assim, em condiçôes de laboratório, o estádio de adaptação de $S$. frugiperda variou de um hospedeiro para outro. Nos hospedeiros estudados em mais de uma época, também se observou variação entre épocas (Tabela 4). Além do milho, o índice de adaptação foi maior que 10 para o sorgo granífero, milheto, sorgo selvagem, capim-marandu e capim-tanzânia. Os hospedeiros em que ocorreram os menores índices de adaptação para
S. frugiperda foram: cana-de-açúcar (1,46 na época II e $2,32$ na época $V)$; grama batatais $(3,00)$ e capim-carrapicho $(3,66)$. Nos demais hospedeiros, o índice de adaptaçáo de $S$. frugiperda foi intermediário.

Os coeficientes de correlação estimados entre o índice de adaptação com a sobrevivência larval $(0,871)$ e com a biomassa de pupas $(0,679)$ foram positivos e com o período larval $(-0,689)$ negativo. Portanto, o índice de adaptação está mais correlacionado com a sobrevivência larval do que com a biomassa de pupas ou período larval.

\section{Índice Relativo de Adaptação (IRA)}

O índice relativo de adaptação foi calculado, para cada hospedeiro e época de avaliação, conforme descrito em Material e Método e estáo apresentados na tabela 5 .

Tabela 4. Índice de Adaptação (IA) de Spodoptera frugiperda, cujas larvas foram alimentadas em diferentes hospedeiros cultivados em cinco épocas, entre novembro de 2006 e setembro de 2008. Sete Lagoas (MG)

\begin{tabular}{|c|c|c|c|c|c|}
\hline \multirow{2}{*}{ Hospedeiro } & \multicolumn{5}{|c|}{ Épocas } \\
\hline & $\mathrm{I}(11-12 / 06)$ & II(03-04/07) & III(07-08/07) & IV(09-10/07) & $V(06-07 / 08)$ \\
\hline Algodão & & 7,2 & & & \\
\hline Amendoim & & 10,2 & & & \\
\hline Cana-de-açúcar & & & 1,5 & & 2,3 \\
\hline Capim-braquiária & & 9,7 & & & \\
\hline Capim-carrapicho & & 3,7 & & & \\
\hline Capim-marandu & & 10,2 & & & \\
\hline Capim-tanzânia & & & 10,5 & & \\
\hline Caruru-de-porco & & & 8,2 & & \\
\hline Grama batatais & & & 3,0 & & \\
\hline Soja & 8,4 & & & & \\
\hline Sorgo granífero & 16,2 & & & & 14,6 \\
\hline Sorgo selvagem & & & 12,6 & & 14,0 \\
\hline Milheto & 14,2 & & & 11,1 & 10,8 \\
\hline Milho & 17,4 & 15,3 & 12,9 & 16,5 & 13,6 \\
\hline Trigo & & & & 9,4 & \\
\hline
\end{tabular}

Tabela 5. Índice Relativo de Adaptação (IRA\%) de Spodoptera frugiperda, cujas larvas foram alimentadas em diferentes hospedeiros cultivados em cinco épocas, entre novembro de 2006 e setembro de 2008. Sete Lagoas (MG)

\begin{tabular}{|c|c|c|c|c|c|}
\hline \multirow{2}{*}{ Hospedeiro } & \multicolumn{5}{|c|}{ Épocas } \\
\hline & $\mathrm{I}(11-12 / 06)$ & II(03-04/07) & III(07-08/07) & IV(09-10/07) & $V(06-07 / 08)$ \\
\hline Algodão & & 47,2 & & & \\
\hline Amendoim & & 66,8 & & & \\
\hline Arroz & 25,0 & & & & \\
\hline Cana-de-açúcar & & & 11,2 & & 17,1 \\
\hline Capim-braquiária & & 63,2 & & & \\
\hline Capim-carrapicho & & 23,9 & & & \\
\hline Capim-marandu & & 66,7 & & & \\
\hline Capim-tanzânia & & & 80,5 & & \\
\hline Caruru-de-porco & & & 63,0 & & \\
\hline Feijão & 47,2 & & & & \\
\hline Grama batatais & & & 23,1 & & \\
\hline Soja & 48,3 & & & & \\
\hline Sorgo granífero & 93,0 & & & & 107,8 \\
\hline Sorgo selvagem & & & 96,7 & & 103,4 \\
\hline Milheto & 81,5 & & & 66,9 & 79,5 \\
\hline Trigo & & & & 57,2 & \\
\hline
\end{tabular}


O índice variou de $11,2 \%$ na cana-de-açúcar a 107,8\% no sorgo granífero. Nota-se que, na época $\mathrm{V}$, o índice relativo de adaptaçấo de $S$. frugiperda, tanto no sorgo granífero, como no sorgo selvagem, foi ligeiramente maior do que no próprio milho. Nas outras épocas, o índice relativo de adaptação de $S$. frugiperda, nesses hospedeiros, foi ligeiramente menor e, se considerarmos as médias das duas épocas, o índice, tanto do sorgo granífero, como do sorgo selvagem, fica em torno dos $100 \%$, indicando que essas diferenças podem estar relacionadas com o erro experimental e não com uma possível interação entre hospedeiro e época de cultivo. Mas, para dirimir esta questão, as interaçóes entre hospedeiros e épocas de cultivo, sobre o estádio de adaptação de $S$. frugiperda, precisam ser reavaliadas nos próximos estudos, incluindo os hospedeiros em todas as épocas de cultivo.

Os coeficientes de correlação entre o índice relativo de adaptaçáo e as variáveis utilizadas no seu cálculo, foram positivos com a sobrevivência $(90,0 \%)$ e com a biomassa de pupas $(71,4 \%)$ e negativo com o período larval $(-56,4 \%)$. Nota-se que o índice relativo de adaptação foi mais correlacionado com a sobrevivência larval do que o índice de adaptação. Nota-se ainda que as correlaçôes do índice relativo de adaptação com a sobrevivência e biomassa de pupas foram aumentadas e com o período larval, reduzidas. Outro aspecto relevante da sobrevivência total das larvas foi sua maior correlaçáo com a sobrevivência durante o desenvolvimento larval $(91,5 \%)$ do que com a sobrevivência inicial (77,0\%). Este resultado sugere maior sensibilidade de larvas maiores, ao efeito dos hospedeiros, do que larvas menores.

Com base no índice relativo de adaptação de cada hospedeiro, pode-se dividir o conjunto de hospedeiros estudado em pelo menos três grupos distintos: aqueles com o índice relativo de adaptação acima de $90 \%$, cuja semelhança ao milho, para a biologia de $S$. frugiperda, os coloca no grupo preferencial; aqueles hospedeiros com o índice no quartil inferior (25\%) e podem ser considerados hospedeiros marginais por ter limitaçôes relevantes à biologia de $S$. frugiperda e, finalmente, aquele grupo intermediário (índice relativo de adaptação entre $26 \%$ e $90 \%$ ), em que está incluída a maioria dos hospedeiros estudados. Esses resultados indicam quais hospedeiros podem ter maior ou menor relevância na dinâmica populacional de $S$. frugiperda no agroecossistema. Não se pode fazer inferência sobre a dinâmica populacional de $S$. frugiperda, exclusivamente com base na adaptação do inseto no hospedeiro, pois, tanto a distribuição, espacial e temporal, desses hospedeiros, como sua densidade no ambiente, são fatores relevantes para a sobrevivência e o desenvolvimento dos insetos.

Os hospedeiros com índice relativo de adaptação alto indicam que, nas condiçóes estudadas (laboratório), suas folhas cortadas são alimentos satisfatórios para S. frugiperda, semelhante às folhas do milho. Pode ser destacado, ainda, que no laboratório, o sorgo granífero testado, cultivar BRS 310, foi o hospedeiro que mais se assemelhou ao milho, para a adaptação de $S$. frugiperda, e a cana-de-açúcar, o hospedeiro menos favorável.

Esse índice pode servir também como referência para selecionar a sequência das culturas a serem usadas na rotação ou sucessão, visando reduzir a população de S. frugiperda em condições de campo, tendo em vista que essa espécie, pelo menos em determinadas condiçôes, possui baixa capacidade de dispersão no campo, cerca de $180 \mathrm{~m}$ (VILARINHo et al., 2006). Essa informação da adaptação de $S$. frugiperda em outros hospedeiros pode ser importante também para os produtores que cultivam o milho Bt. É recomendado o uso de área de refúgio como estratégia para o manejo da resistência do inseto às proteínas $\mathrm{e}$, assim, o conhecimento do estádio de adaptação de $S$. frugiperda, em outros hospedeiros, pode contribuir para a escolha de culturas alternativas nas proximidades das lavouras Bt's, aumentando ou diminuindo a área de refúgio.

$O$ índice relativo de adaptação pode também ser usado nos estudos de variabilidade populacional de S. frugiperda, tanto em um mesmo espaço físico em culturas diferentes, conforme os trabalhos realizados por Busato et al. (2004), ou em função da variação espacial, através da avaliação de populaçôes oriundas de diferentes regiôes. Pode, ainda, ser estimado ao longo do tempo para se avaliar a evoluçáo da adaptaçáo de espécies de insetos a um ou mais hospedeiros. Pogue (1995) cita mais de cem espécies de plantas hospedeiras de $S$. frugiperda, portanto, esta espécie exibe alto grau de polifagia, podendo se alimentar e sobreviver em diversos grupos de plantas. Por esse motivo, é natural que $S$. frugiperda proporcione diferentes estádios de adaptação, em função da disponibilidade de hospedeiros no ambiente onde ela está se reproduzindo. Respostas diferenciais de $S$. frugiperda a diferentes hospedeiros foram observadas também por SÁ et al. (2009). Mesmo em hospedeiro preferencial como o milho (Cruz, 1995), pode-se observar respostas diferentes, em função do genótipo, como registrado por Mendes et al. (2011). Evidentemente, os mecanismos de resistência de plantas a insetos interferem com a adaptação das espécies nos seus respectivos hospedeiros. Assim, tanto a sobrevivência como o período larval e a biomassa de pupas são variáveis afetadas, basicamente, pela qualidade alimentar da planta para o inseto ou pela presença de substâncias secundárias, com propriedades defensivas para a planta (Painter, 1951). Ainda, segundo esse autor, esses efeitos podem ser manifestados através da não preferência para alimentação ou pela antibiose. O mecanismo de tolerância é uma resposta da evolução adaptativa da planta ao inseto, cujos danos não afetam sua sobrevivência e produção econômica. A não preferência para alimentação está relacionada com a morfologia e consistência dos tecidos, mas, principalmente, pela presença de inibidores de alimentação, que podem prejudicar o desenvolvimento dos insetos 
herbívoros (Santos e Boiça Junior, 2001; Willians et al., 1990; Willians e Beckley, 1992). Portanto, métodos práticos e eficazes para comparar esses diferentes hospedeiros e, assim, definir o papel de cada um na dinâmica populacional das espécies de insetos herbívoros são da mais alta relevância para definir estratégias de manejo de pragas e da resistência de insetos aos transgênicos.

\section{CONCLUSÃO}

Em laboratório, as principais variáveis biológicas de S. frugiperda, como sobrevivência, período larval e biomassa de pupas, são afetadas pelas espécies de hospedeiros e pela época de cultivo desses hospedeiros.

O Índice Relativo de Adaptação (IRA) é um indicador satisfatório do estádio de adaptação de $S$. frugiperda em diferentes hospedeiros e, das três variáveis analisadas, a sobrevivência dos insetos é a que mais afeta esse índice.

No sorgo granífero e no sorgo selvagem, $S$. frugiperda possui estádio de adaptação muito próximo ao do milho. No arroz, capim-carrapicho, grama batatais e na cana-de-açúcar, S. frugiperda tem estádio de adaptação baixo. Nos hospedeiros milheto, capim-tanzânia, amendoim, capim-marandu, capim-braquiária, caruru-de-porco, trigo, soja, algodáo e feijão, o estádio de adaptação de $S$. frugiperda é intermediário;

\section{AGRADECIMENTOS}

Os autores expressam seus agradecimentos à Embrapa Milho e Sorgo e à Universidade Federal de Minas Gerais, pelo apoio logístico; ao Conselho Nacional de Pesquisa e Desenvolvimento $(\mathrm{CNPq})$ e à Fundação de Amparo à Pesquisa do Estado de Minas Gerais (FAPEMIG), pelo apoio financeiro. Também, aos funcionários da Embrapa Milho e Sorgo, Eustáquio Francisco Souza de Oliveira e Mauro Eugênio de Resende Paulinelli, às estagiárias, Talita Costa Fermino, Sabrina Cordeiro de Macedo e Yara Lopes Xavier, pela colaboração na realização dos trabalhos de laboratório e campo.

\section{REFERÊNCIAS}

BARAH, A.; SENGUPTA, A.K. Correlation and regression studies between pupal weight and fecundity of muga silkworm Antheraea assama Westwood (Lepidoptera: Saturniidae) on four different foodplants. Acta Physiologica Hungarica, v.78, p.261-264, 1991.

BAVARETO, A.; GARCIA, M.S.; GRÜTZMACHER, A.D.; FORESTI, J.; RINGENBERG, R. Biologia comparada de Spodoptera cosmioides (Walk.) (Lepidoptera: Noctuidae) em cebola, mamona, soja e feijão. Ciência Rural, v.33, p.993-998, 2003.

BUSATO, G.R.; GRÜTZMACHER, A.D.; GARCIA, M.S.; GIOLO, F.P.; STAFANELLO, JR.G.J.; ZOTTI, M.J. Preferência para Alimentação de Biótipos de Spodoptera frugiperda (J.E. Smith, 1797) (Lepidoptera: Noctuidae) por Milho, Sorgo, Arroz e CapimArroz. Revista Brasileira de Agrociência, v.10, p.215-218, 2004.

CORTEZ, M.G.R.; WAQUIL, J.M. Influência de cultivar e nível de infestação de Spodoptera frugiperda (J. E. Smith) (Lepidoptera: Noctuidae) no rendimento de gráos. Anais da Sociedade Entomológica do Brasil, v.26, p.407-410, 1997.

CRUZ, I.; TURPIN, F.T. Yield impact of larval infestation of the fall armyworm (Lepidoptera: Noctuidae) to midwhorl stage of corn. Journal of Economic Entomology, v.76, p.1052-1054, 1983.

CRUZ, I. Manejo integrado de pragas de milho com ênfase para o controle biológico. In: CICLO DE PALESTRAS SOBRE O CONTROLE BIOLÓGICO DE PRAGAS, 4., 1995, Campinas, SP. Anais... Campinas: SEB/Instituto Biológico, 1995. p.48-92.

CTNBio. Parecer técnico conclusivo consolidado e final para liberação comercial, 2011. Disponível em: www.ctnbio.gov.br/ upd_blob/0001/1543.doc. Acesso em 2013.

FERREIRA, D.F. Sisvar versão 4.2. Lavras: UFLA, 2003.

IBGE. Instituto Brasileiro de Geografia e Estatística. Estatística da agropecuária. Disponível em: http://www.ibge.gov.br/home/ estatistica/indicadores/agropecuaria/lspa/default.shtm. 2009.

LEUCK, D.B.; PERKINS, W.D. A method of estimating a fall armyworm progeny reduction when evaluating control achievement by host-plant resistance. Journal of Economic Entomology, v.65, p.482-483, 1972.

MEAGHER, R.L.; NAGOSHI, R.N.; STUHL, C.; MITCHELL, E.R. Larval development of fall armyworm (Lepidoptera: Noctuidae) on different cover crop plants. Florida Entomologist, v.87, p.454-460, 2004.

MENDES, S.M. ; BOREGAS, K.G.B. ; LOPES, M.E ; WAQUIL, J.M. ; WAQUIL, M.S. Respostas da lagarta-do-cartucho ao milho geneticamente modificado expressando a toxina Cry $1 \mathrm{~A}(\mathrm{~b})$. Pesquisa Agropecuária Brasileira, v.46, p.239-244, 2011.

MOSCARDI, F.; BARFIELD, C.S.; ALLEN, G.E. Impact of soybean phenology on velvetbean caterpillar (Lepidoptera: Noctuidae): oviposition, egg hatch, and adult longevity. Canadian Entomology, v.113, p.113-119, 1981.

MURÚA, G.; VIRLA, E. Population parameters of Spodoptera frugiperda (J.E. Smith) (Lep.: Noctuidae) fed on corn and two predominant grass in Tucuman (Argentina). Acta Zoológica Mexicana, v.20, p.199-210, 2004.

PAINTER, R. Insect resistance in crop plants. New York: Macmillan, 1951. 520p.

PANIZZI, A.R.; OLIVEIRA, L.J.; SILVA, J.J. Survivorship, larval development and pupal weight of Anticarsia gemmatalis (Hübner) (Lepidoptera: Noctuidae) feeding on potential leguminous host plants. Neotropical Entomology, v.33, p.563-567, 2004.

PENCOE, N.L.; MARTIN, P.B. Development and reproduction of fall armyworms on several wild grasses. Environmental Entomology, v.10, p.999-1002, 1981. 
PENCOE, N.L.; MARTIN, P.B. Fall armyworm (Lepidoptera: Noctuidae) larval development and adult fecundity on five grass hosts. Environmental Entomology, v.11, p.720-724, 1982.

POGUE, M.G. World Spodoptera Database (Lepidoptera: Noctuidae), 1995. Disponível em: http://www.sel.barc.usda.gov/ lep/spodoptera/spodoptera.html. Acesso em 2007.

SÁ, V.G.M.; FONSECA, B.V.C.; BOREGAS, K.G.B.; WAQUIL, J.M. Sobrevivência e desenvolvimento larval de Spodoptera frugiperda (J.E. Smith) (Lepidoptera: Noctuidae) em hospedeiros alternativos. Neotropical Entomology, v.38, p.108-115, 2009.

SANTOS, T.M.; BOIÇA JUNIOR, A.L. Resistência de genótipos de algodoeiro (Gossypium hirsutum L.) a Alabama argillacea (Hübner) (Lepidoptera: Noctuidae). Neotropical Entomology, v.30, p.297-303, 2001.
VILARINHO, E.C.; FERNANDES, O.A.; OMOTO, C.; HUNT, T.E. Oil-Soluble Dyes for Marking Spodoptera frugiperda (Lepidoptera: Noctuidae). Journal of Economic Entomology, v.99, p.2110-2115, 2006.

WAQUIL, J.M.; VILELLA, F.M.F.; FOSTER, J.E. Resistência do milho (Zea mays 1.) transgênico (Bt) à lagarta-do-cartucho, Spodoptera frugiperda (J. E. Smith) (Lepidoptera: Noctuidae). Revista Brasileira de Milho e Sorgo, v.3, p.1-11, 2002.

WILLIAMS, W.P.; DAVIS, F.M. Registration of Mp705, Mp706 and Mp707 germplasm lines of maize. Crop Science, v.24, p.1217, 1984 .

WILLIANS W.P.; BUCKLEY, P.M. Growth to fall armyworm (Lepidoptera: Noctuidae) larvae on resistant and susceptible corn. Journal of Economic Entomology,, v.83, p.2039-2042, 1992. 